\title{
Actions combinées de Eichhornia crassipes et Pistia stratiotes pour traitement tertiaire de l'effluent des bassins facultatifs de la station d'épuration de Buterere, Burundi
}

\author{
Pierre NTAKIYIRUTA ${ }^{1,2 *}$, Gaston NSAVYIMANA ${ }^{2}$, Bi Gouessé Henri BRITON ${ }^{1}$, \\ Kopoin ADOUBY ${ }^{1}$, David NAHIMANA ${ }^{2}$ et Gaspard NTAKIMAZI ${ }^{2}$
}
${ }^{1}$ Laboratoire des Procédés Industriels de Synthèse, de l'Environnement et des Energies Nouvelles (LAPISEN), Institut National Polytechnique Félix Houphouët Boigny, BP 1093 Yamoussoukro, Côte d'Ivoire.
${ }^{2}$ Centre de Recherche en Sciences Naturelles et de l'Environnement (CRSNE), Université de Burundi, BP 1550 Bujumbura, Burundi.
*Auteur correspondant; E-mail: pierrentakiyiruta@yahoo.fr; Tél.: 0025779229032

\section{RESUME}

Le lac Tanganyika, exutoire des eaux de rejet de la station d'épuration de Buterere, est menacé par l'eutrophisation à cause de l'enrichissement en nutriments. Conséquence de la pression démographique, un redimensionnement en vue d'accroître la performance épuratrice de ladite station nécessiterait d'importants investissements. Fort de cela, l'action combinée de Pistia stratiotes et Eichhornia crassipes en traitement tertiaire a été envisagée. A cet effet, six tanks disposés deux à deux en série ont constitué des bassins de phytoremediation contre deux témoins. Ces tanks étaient alimentés en continu par l'effluent prélevé à la sortie des bassins facultatifs de la station. Les Pistia stratiotes et Eichhornia crassipes étaient respectivement cultivées dans ces tanks placés en amont et aval du pilote expérimental avec une densité initiale de 60 pieds $/ \mathrm{m}^{2}$. Ces essais ont été suivis tous les deux jours à la sortie de chaque tank en évaluant les concentrations des paramètres jusqu'à atteindre une efficacité de $86,08 \% ; 57,37 \%, 46,21 \% ; 57,41 \%, 74,36 \% ; 99,37 \% ; 93,77 \%$ et $100 \%$ respectivement pour $\mathrm{NH}_{4}{ }^{+}$, $\mathrm{NO}_{3}{ }^{-}, \mathrm{PO}_{4}{ }^{3-}, \mathrm{DCO}, \mathrm{DBO}$, Chlorophylle A, Chlorophylle B et Chlorophylle C. Au vue de ces résultats, cette action combinée est une alternative pour renforcer la capacité épuratrice de la station de Buterere.

(C) 2020 International Formulae Group. All rights reserved.

Mots clés : Eaux usées, nutriments, phytoremédiation, lagunage, tank, mode continu.

\section{Combined actions of Eichhornia crassipes and Pistia stratiotes for tertiary treatment of the effluent from the facultative basins of the Buterere treatment plant, Burundi}

\begin{abstract}
Lake Tanganyika, the outlet for the Buterere sewage treatment plant, is threatened by eutrophication due to nutrient enrichment. As a consequence of population pressure, a resizing to increase the treatment performance of the plant would require significant investments. With this in mind, the combined action of Pistia stratiotes and Eichhornia crassipes in tertiary treatment was considered. For this purpose, six tanks arranged two by two in series constituted phytoremediation basins against two controls. These tanks were continuously fed by the
\end{abstract}


effluent taken at the exit of the optional basins of the station. Pistia stratiotes and Eichhornia crassipes were respectively cultivated in these tanks placed upstream and downstream of the experimental pilot with an initial density of $60 \mathrm{feet} / \mathrm{m}^{2}$. These tests were followed every two days at the exit of each tank by evaluating the concentrations of the parameters until an efficiency of $86.08 \% ; 57.37 \%, 46.21 \% ; 57.41 \%, 74.36 \% ; 99.37 \%$; $93.77 \%$ and $100 \%$ respectively for $\mathrm{NH}_{4}{ }^{+}, \mathrm{NO}_{3}{ }^{-}, \mathrm{PO}_{4}{ }^{3-}, \mathrm{COD}, \mathrm{BOD}$, Chlorophyll $\mathrm{A}$, Chlorophyll $\mathrm{B}$ and Chlorophyll $\mathrm{C}$ was reached. In view of these results, this combined action is an alternative to strengthen the scrubbing capacity of the Buterere plant.

(C) 2020 International Formulae Group. All rights reserved.

Keywords: Wastewater, nutrients, phytoremediation, lagooning, tank, continuous mode.

\section{INTRODUCTION}

Avec le développement galopant de l'urbanisation et de l'industrialisation, le volume des eaux usées domestiques et industrielles ne cesse d'augmenter. La gestion quotidienne de ces eaux usées devient de plus en plus une préoccupation majeure des pays en développement qui doivent consentir un budget conséquent pour assainir le cadre de vie (Yahiaoui et al., 2018). En effet, les eaux usées sont généralement le nid des bactéries responsables des maladies hydriques et des nuisances olfactives lorsqu'elles ne sont pas traitées correctement. Au nombredes procédés de traitement, le lagunage naturel constitue une technologie efficace à moindre coût et le mieux adapté pour les pays en développement à climat tropical (Kone, 2002). Toutefois, un des défis à relever pour ce système de traitement est l'élimination des nutriments qui causent toujours un véritable problème compte tenu des rejets qui ne respectent pas des normes (Mama et al., 2012; Wang and Sample, 2014; Gboko et al., 2019).

C'est le cas de la station d'épuration (STEP) de Buterere (Bujumbura / Burundi) de type lagunage naturel qui est dans l'incapacité d'éliminer convenablement les nutriments à cause de l'absence de traitement tertiaire (Nsavyimana, 2015; Buhungu et al., 2017). En outre, cette station qui avait été conçue en 1994 pour traiter $40000 \mathrm{~m}^{3} / \mathrm{j}$ (Nsavyimana, 2015), doit désormais en traiter plus à cause de la croissance démographique estimée à 2,4\% (Sindayigaya, 2015). Par conséquent, l'étude de la qualité des eaux de la rivière Kinyankonge, exutoire des rejets de la STEP de Buterere/Burundi et affluent du lac Tanganyika, a montré que cette rivière est contaminée en nutriments par ces eaux de rejet (Buhungu et al., 2018).

Par ailleurs, des études ont montré que la phytoremédiation au moyen des plantes aquatiques telles que Eichhornia crassipes (E. crassipes)et Pistia stratiotes (P. stratiotes) est une technique très adaptée pour l'élimination des polluants dans les eaux usées, y compris les nutriments (Shah et al., 2015; Victor et al., 2016; Qin et al., 2016; Lu et al., 2018; Ting et al,. 2018).Les résultats de ces différentes études précitées ont montré que ces plantes aquatiques éliminent les métaux lourds et les composés organiques ainsi que les différentes formes des composés azotés et phosphatés. De ces études réalisées antérieurement, quelquesunes seulement se sont intéressées à faire des investigations comparatives sur l'efficacité d'élimination des polluants dans les eaux usées par E. crassipes et P. stratiotes (Ismail et al., 2014; Qin et al., 2016; Victor et al., 2016; Zimmels et al., 2019). Il ressort de ces travaux, des conclusions divergentes car pendant que certains rapportent que E. crassipes est efficace pour l'azote et $P$. stratiotes pour le phosphore (Qin et al., 2016; Victor et al., 2016), d'autres trouvent que c'est le contraire(Gupta et al., 2012). Ces études ont été réalisées, pour la plupart, en mode d'alimentation discontinu dans des dispositifs d'expérimentation. Par conséquent, ces résultats seraient difficiles à extrapoler sur des systèmes de traitement continu opérant dans des conditions réelles.

Au meilleur de nos connaissances, peu d'études sur la phytoremédiation dans le système lagunage naturel incluant l'action combinée de différentes plantes aquatiques et fonctionnant sous climat tropical d'Afrique sub-saharienne ont été réalisées. A cet effet, 
une hypothèse de recherche selon laquelle l'action combinée d'E. crassipes et $P$. stratiotes permettrait d'éliminer convenablement les nutriments dans un système lagunage naturel après le bassin facultatif. De fait, le bassin de maturation qui assurait le traitement tertiaire serait substitué par les bassins à macrophytes. Cela pourrait être possible en prenant en compte des facteurs clés comme le temps de séjour hydraulique (TSH) pour réaliser de meilleures performances épuratoires (Mara, 2003).

Cette étude visait donc à évaluer la synergie de E. crassipes et $P$. stratiotes pour éliminer les nutriments, dans le système lagunage naturel fonctionnant sous des conditions précitées lorsque ces plantes aquatiques sont utilisées séparément. Ainsi, cette étude propose: (i) une nouvelle conception du bassin de maturation consistant à le subdiviser en deux sous-bassins et dans lesquels $E$. crassipes et $P$. stratiotes sont introduits dans des conditions bien précises; et (ii) de travailler en mode d'alimentation continue en effluent pour simuler la réalité de terrain.

\section{MATERIEL ET METHODES}

\section{Dispositif expérimental}

Le lagunage naturel par phytoremédiation a été réalisé sur un pilote expérimental installé au Campus Mutanga de $1^{\prime}$ Université du Burundi (Coordonnées GPS : S322'54", E29²3'03" ; altitude: 798,7 m). La zone d'étude est caractérisée par une température moyenne annuelle de $24,1^{\circ} \mathrm{C}$ avec une température variant de 23 à $28^{\circ} \mathrm{C}$ et une pluviométrie comprise entre 800 et 1100 $\mathrm{mm} / \mathrm{an}$ (Nsavyimana, 2015).

E. crassipes et $P$. stratiotes qui sont des macrophytes flottants, ont été choisis pour assurer le traitement tertiaire d'une eau issue d'un traitement par lagunage naturel. De ce fait, le bassin a été subdivisé en deux sous bassins interconnectés et dans lesquels $P$. stratiotes et $E$. crassipes sont simultanément cultivées. Pour évaluer la reproductibilité des essais, six tanks expérimentaux (P1 - P2 $\mathrm{P} 3 / / \mathrm{E} 1-\mathrm{E} 2$ - E3) et deux tanks témoins (T1 // T2) ont constitué le pilote expérimental y compris le grand tank réservoir (capacité $5 \mathrm{~m}^{3}$ ) à eau usée sur une superficie de $1200 \mathrm{~m}^{2}$ (Figure 1). Ces tanks ont chacun une capacité de $1,5 \mathrm{~m}^{3}$ (1,68 $\mathrm{m} \mathrm{X} \mathrm{1,38} \mathrm{m} \mathrm{X} \mathrm{0,65} \mathrm{m).} \mathrm{Les}$ tanks T1, P1, P2 et P3 sont connectés chacun au tank réservoir par un répartiteur pour recevoir progressivement de l'eau usée en mode continu. Par contre, les tanks T2, E1, E2 et $\mathrm{E} 3$ sont connectés respectivement à $\mathrm{T} 1, \mathrm{P} 1$, $\mathrm{P} 2$ et $\mathrm{P}$ 3. C'est donc à partir des tanks $\mathrm{P} 1, \mathrm{P} 2$ et $\mathrm{P} 3$ des P. stratiotes en passant par les tanks E1, E2 et E3 des E. crassipes que les eaux phytoépurées sont rejetées du système après un temps de séjour hydraulique de 16,6 jours (soit 8,3 jours/tank). En effet, un débit de $0,27 \mathrm{~m}^{3} / \mathrm{j}$ d'eaux usées sont évacuées dans chaque tank à partir du tank réservoir. Pour conserver ce débit, le tank réservoir est aussi alimenté tous les 5 jours en eaux usées prélevées à la sortie du bassin facultatif de la STEP de Buterere (S $3^{\circ} 20^{\prime} 16,647^{\prime \prime}$; E $29^{\circ} 15^{\prime} 32,525^{\prime \prime}$; et altitude de $762,5 \mathrm{~m}$ ). Les eaux usées qui séjournent dans les deux tanks $\mathrm{T} 1$ et $\mathrm{T} 2$ sortent sans être mélangées à celles des tanks à macrophytes. Ces tanks $\mathrm{T} 1$ et $\mathrm{T} 2$ ont été utilisés pour mettre en évidence uniquement l'action des microorganismes et des microphytes de l'effluent brut. La Figure 1 illustre le schéma du pilote expérimental utilisé pour mener cette étude.

\section{Macrophytes et conditions expérimentales}

En considérant, les conditions optimales obtenues par Mahunon et al. (2018), la densité des plantes a été fixée à 144 pieds par espèce sur 2,4 $\mathrm{m}^{2}$ du plan d'eau de chaque tank pour démarrer la phytoremédiation. Ces macrophytes frais et vigoureux ayant pratiquement la même taille ont été collectés dans le bassin versant de la rivière Kinyankonge, exutoire des rejets de la STEP de Buterere/Bujumbura.

\section{Evaluation de l'efficacité de traitement}

La qualité de l'eau usée prélevée à la sortie du bassin facultatif de la STEP a été à chaque fois évaluée au cours des dix prélèvements par la mesure $\mathrm{du} \mathrm{pH}$, de la Chlorophylle (A, B et C), du phosphore $\left(\mathrm{PO}_{4}{ }^{3-}\right.$ ), de l'azote ammoniacal $\left(\mathrm{N}-\mathrm{NH}_{4}{ }^{+}\right)$, de l'azote 
nitrique $\left(\mathrm{N}^{-\mathrm{NO}_{3}}{ }^{-}\right)$, de la demande chimique en oxygène (DCO) et de la demande biochimique en oxygène (DBO). Ce système lagunage naturel comporte une dernière étape ou bassin de maturation devant réceptionner les eaux issues du bassin facultatif pour assurer le traitement tertiaire. Les mêmes paramètres indicateurs de pollution ont été également analysés sur des échantillons prélevés tous les deux jours, à l'entrée et à la sortie des tanks. A part le $\mathrm{pH}$ qui a été déterminé par un $\mathrm{pH}$-mètre de type WTW 340i, tous les autres paramètres ont été obtenus selon les normes françaises AFNOR (1994). Tous ces résultats ont été exploités pour déterminer le rendement de la phytoremédiation par ces deux macrophytes sur ce pilote expérimental. Les moyennes et les écarts types ont été également calculés pour mieux apprécier la variation des données par rapport aux deux séries à trois tanks chacune. Ce rendement a été calculé selon l'expression suivante :

$$
Y(\%)=\left(1-\frac{C_{f}}{C_{i}}\right) * 100
$$

Où $Y, C_{i}$ et $C_{f}$ représentent respectivement le rendement de la phytoremédiation (\%), les concentrations initiale et finale des paramètres indicateurs de pollution.

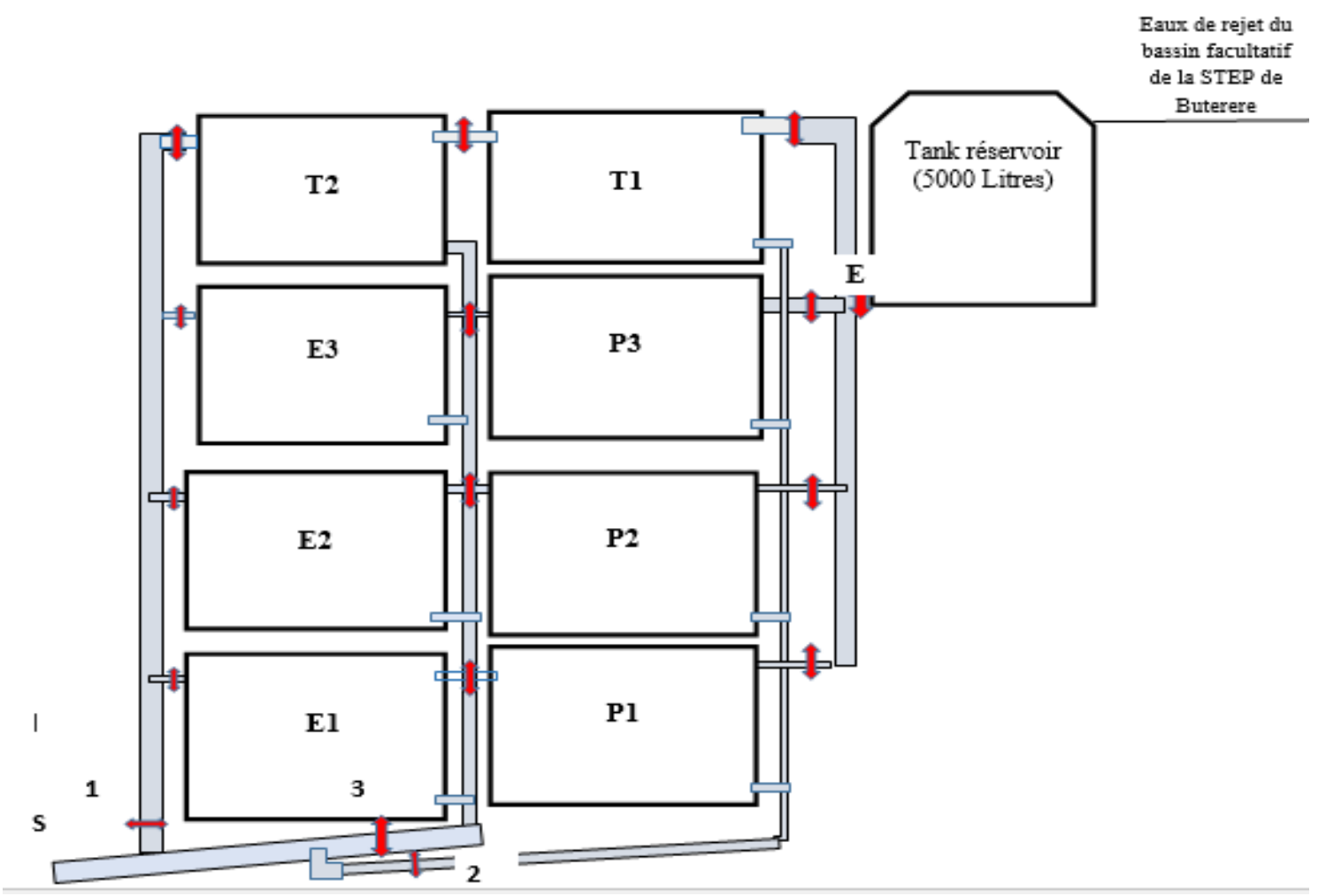

$\boldsymbol{E}:$ Effluent à traiter ; P. stratiotes (P1 : essai $1 ; \boldsymbol{P 2}$ : essai 2, P3 : essai 3); E. crassipes (E1 : essai $1 ; \boldsymbol{E} 2$ : essai $2, \boldsymbol{E} 3$ : essai 3), $\mathbf{S}$ : Sortie d'effluent traité ; 1 : Evacuation de l'effluent traité ; 2 : Evacuation P1, P2 et P3 à la fin des essais ; 3 : Evacuation E1; E2 et E3 à la fin des essais; T1 et T2 : Tanks témoins, Vanne :

Figure 1 : Schéma du pilote expérimental de la phytoremédiation en mode continu. 


\section{RESULTATS}

\section{Caractéristiques de l'effluent alimentant le pilote expérimental}

Les caractéristiques moyennes de l'effluent du bassin facultatif sont présentées dans le Tableau 1. Ces résultats ont été comparés à la qualité de l'effluent du bassin de maturation de la station d'épuration de Buterere (Nsavyimana, 2015) et aux données relatives à la norme de rejet des eaux usées selon l'OMS (Carr et al., 2004). En effet, l'effluent du bassin de maturation correspond à la dernière étape de traitement de la station d'épuration (STEP) de Buterere. De toute évidence, on constate que l'effluent du bassin facultatif est chargé par rapport aux eaux de rejet de la dite STEP. Par contre, les caractéristiques des eaux issues de la STEP ne sont pas en conformité avec les valeurs limites préconisées par l'OMS (Carr et al., 2004). Cela traduit les limites de cette STEP qui rejette des eaux susceptibles de polluer les cours d'eau. Par ailleurs, si l'élimination de la pollution organique évaluée par la DCO et la DBO semble satisfaisante, force est de reconnaître que ce procédé est peu efficace vis-à-vis des nutriments (phosphore et azote). Par conséquent, la mise en place d'un traitement tertiaire par la phytoremédiation utilisant les actions combinées des $P$. stratiotes et $E$. crassipes, pourrait trouver son intérêt dans cette étude à travers l'évolution des paramètres de pollution.

\section{Evolution des paramètres de pollution}

La performance du dispositif mis en place a été suivie par les cinétiques de réduction de $\mathrm{PO}_{4}{ }^{3-}$ (Figure 2), de $\mathrm{NH}_{4}{ }^{+}$(Figure 3 ), de $\mathrm{NO}_{3}{ }^{-}$(Figure 4) et des Chlorophylles (Figure 5). A cet effet, une réduction significative de $\mathrm{PO}_{4}{ }^{3-}$ (Figure 2) est constatée après un temps de séjour de l'effluent successivement dans les bassins à $P$. stratiotes puis à $E$. crassipes. Par ailleurs, pour le bassin témoin, une légère diminution de $\mathrm{PO}_{4}{ }^{3-}$ est certainement due à l'action des microphytes ou des algues. On constate qu'après $25^{\mathrm{e}}$ jour, une remontée de la concentration de $\mathrm{PO}_{4}{ }^{3-}$ qui pourrait traduire le vieillissement et la mort des macrophytes. En effet, la décomposition des macrophytes dans l'eau suite à cette mortalité entraîne l'enrichissement du milieu en nutriments et en composés organiques. De ce fait, un renouvèlement des plantes doit s'imposer pour continuer la phytoremédiation avec de jeunes plantes. Ainsi, en considérant un séjour maximal de 25 jours, on réalise un rendement de $46,21 \%$ de réduction de $\mathrm{PO}_{4}{ }^{3-}$.

En ce qui concerne l'élimination de $\mathrm{NH}_{4}{ }^{+}$, celle-ci est passée de 82,8 à $4,32 \mathrm{mg} / \mathrm{L}$ après l'activité des macrophytes (Figure 3 ). En effet, l'élimination de $\mathrm{NH}_{4}{ }^{+}$a été remarquable jusqu'au $13^{\mathrm{e}}$ jour avec un taux de $94,78 \%$ à la sortie du dispositif expérimental. En outre, on remarque que la cinétique d'élimination de $\mathrm{NH}_{4}{ }^{+}$est pratiquement similaire au niveau des deux macrophytes.

L'un des résultats aussi attendu est la cinétique d'élimination du nitrate. Ainsi, à travers la Figure 4, on constate une chute rapide de la concentration de $\mathrm{NO}_{3}{ }^{-}$au bout de 15 jours malgré l'apport continu de la transformation biologique de $\mathrm{NH}_{4}{ }^{+}$en $\mathrm{NO}_{3}{ }^{-}$et celui fourni par l'eau brute.

Quant à la cinétique d'élimination des chlorophylles, les résultats ont montré que la charge en Chlorophylle A (Figure 5a) augmente de 886 à 1304,36 $\mu \mathrm{g} / \mathrm{L}$ jusqu'au $15^{\mathrm{e}}$ jour dans les bassins à $P$. stratiotes et $E$. crassipes, puis celle en Chlorophylle $\mathrm{C}$ (Figure 5c) atteint le maximum à $616,60 \mu \mathrm{g} / \mathrm{L}$ au $6^{\mathrm{e}}$ jour. Cette tendance est pratiquement la même dans les bassins témoins et même dans le tank à eau brute.

\section{Efficacité du pilote expérimental}

Après avoir présenté la cinétique d'élimination de certains paramètres de pollution, il est important d'évaluer l'efficacité du dispositif expérimental à travers les taux d'abattement (Tableau 2). Cela nous permet de comparer ces résultats à la qualité de l'effluent à la sortie de la STEP de Buterere et des tanks témoins. A cet effet, cette performance du pilote expérimental a été scindée sur deux périodes au regard des cinétiques d'élimination. Il s'agit des 15 premiers jours et le $31^{\mathrm{e}}$ jour de la phytoremédiation. Pour les nutriments, des rendements de $91,1 \%, 57,36 \%$ et $32,61 \%$ respectivement pour $\mathrm{NH}_{4}{ }^{+}, \mathrm{NO}_{3}{ }^{-}$et 
$\mathrm{PO}_{4}{ }^{3-}$ après 15 jours ont été obtenus. Ces résultats traduisent une bonne activité des macrophytes comparativement aux données obtenues au niveau des tanks témoins. Cette période correspond à une croissance accélérée des macrophytes à tel enseigne que la demande en nutriment est de plus en plus forte.

Cependant, jusqu'au $31^{\mathrm{e}}$ jour, on assiste à une augmentation de la production des nitrates déjà révélée précédemment. Par contre, cette tendance observée pour les nutriments est vraisemblablement contraire chez les Chlorophylles car au $15^{\mathrm{e}}$ jour la production est remarquable avec des concentrations bien supérieures à celles de l'effluent brut. Mais après cette période, la croissance harmonieuse des macrophytes a renversé la tendance au point d'avoir des rendements pouvant atteindre $100 \%$.

Tableau 1 : Caractéristiques des eaux d'alimentation (Effluent des bassins facultatifs) du pilote expérimental.

\begin{tabular}{|c|c|c|c|}
\hline Paramètre & $\begin{array}{l}\text { Effluent des bassins } \\
\text { facultatifs }\end{array}$ & $\begin{array}{c}\text { Rejets de la STEP } \\
\text { Buterere }\end{array}$ & $\begin{array}{c}\text { Limites de rejet } \\
\text { (OMS) }\end{array}$ \\
\hline $\mathrm{pH}$ & $8,3 \pm 0,12$ & $8,2 \pm 0,13$ & $6,5-9,5$ \\
\hline Chlorophylle A $(\mu \mathrm{g} / \mathrm{L})$ & $8860 \pm 90,0$ & $6125 \pm 101,0$ & $0-200$ \\
\hline Chlorophylle B ( $\mu \mathrm{g} / \mathrm{L})$ & $640 \pm 10$ & $424,30 \pm 9,55$ & $0-200$ \\
\hline Chlorophylle C ( $\mu \mathrm{g} / \mathrm{L})$ & $1530 \pm 9.8$ & $1280,10 \pm 8,18$ & $0-200$ \\
\hline $\mathrm{PO}_{4}{ }^{3-}(\mathrm{mg} / \mathrm{L})$ & $28,11 \pm 2,15$ & $25,40 \pm 3,13$ & $\leq 2$ \\
\hline $\mathrm{N}-\mathrm{NH}_{4}{ }^{+}(\mathrm{mg} / \mathrm{L})$ & $82,80 \pm 0,25$ & $68,50 \pm 10,89$ & $\leq 15$ \\
\hline $\mathrm{N}-\mathrm{NO}_{3}{ }^{-}(\mathrm{mg} / \mathrm{L})$ & $47,03 \pm 1,17$ & $41,20 \pm 4,71$ & $\leq 30$ \\
\hline $\mathrm{DCO}\left(\mathrm{mg} \mathrm{O}_{2} / \mathrm{L}\right)$ & $358,13 \pm 30,5$ & $123,00 \pm 17,92$ & $\leq 150$ \\
\hline $\mathrm{DBO}_{5}\left(\mathrm{mg} \mathrm{O}_{2} / \mathrm{L}\right)$ & $260,00 \pm 20,45$ & $51,00 \pm 6,98$ & $\leq 50$ \\
\hline
\end{tabular}

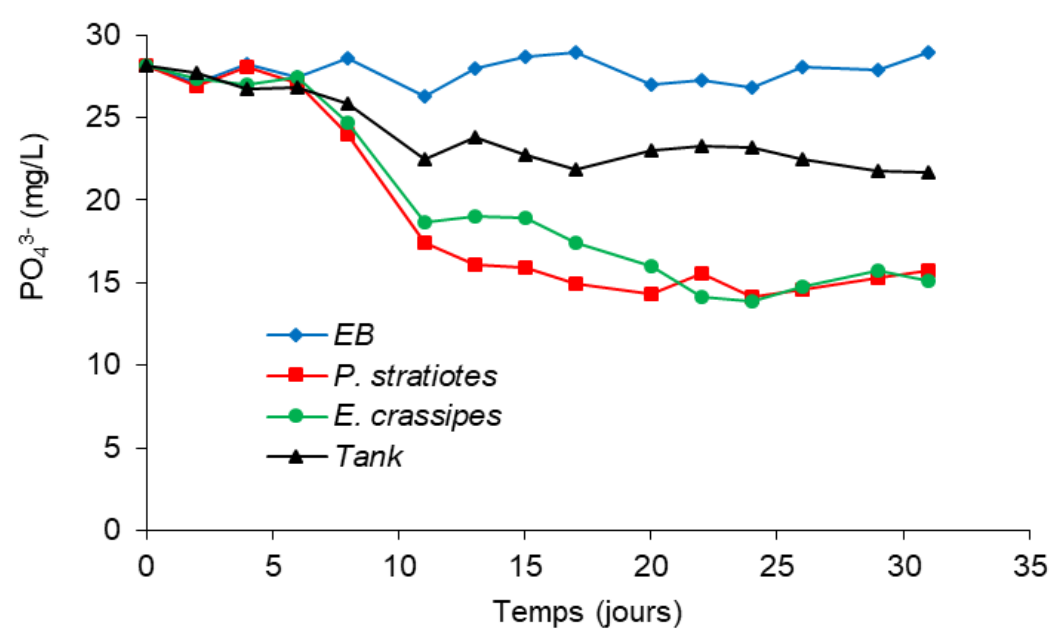

Figure 2 : Cinétique de réduction de $\mathrm{PO}_{4}{ }^{3-}$ dans le dispositif expérimental. 


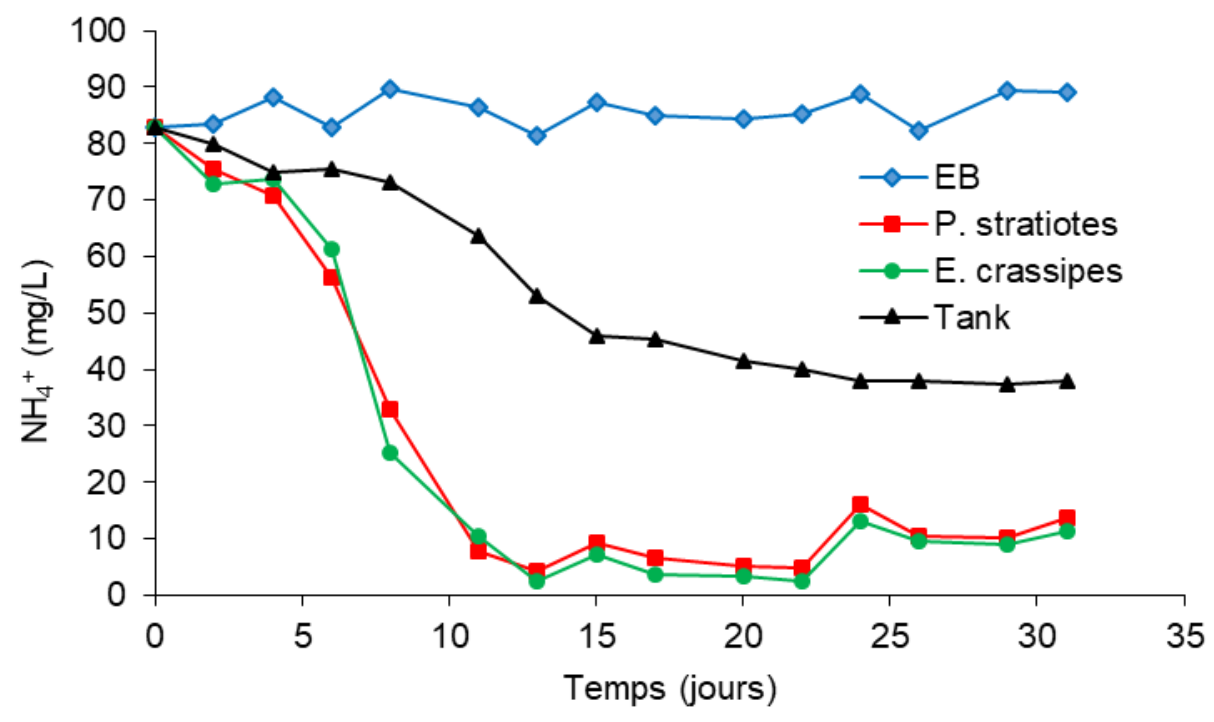

Figure 3 : Cinétique de réduction de $\mathrm{NH}_{4}{ }^{+}$dans le dispositif expérimental.

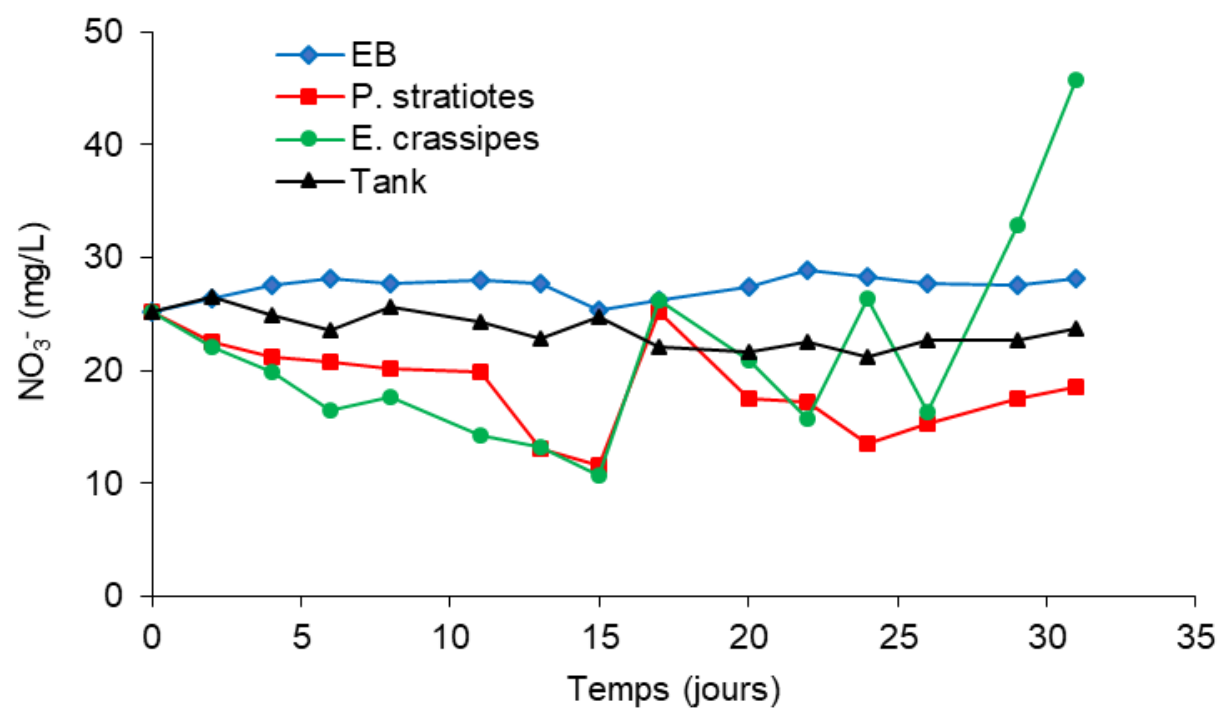

Figure 4 : Cinétique de réduction de $\mathrm{NO}_{3}{ }^{-}$dans le dispositif expérimental. 

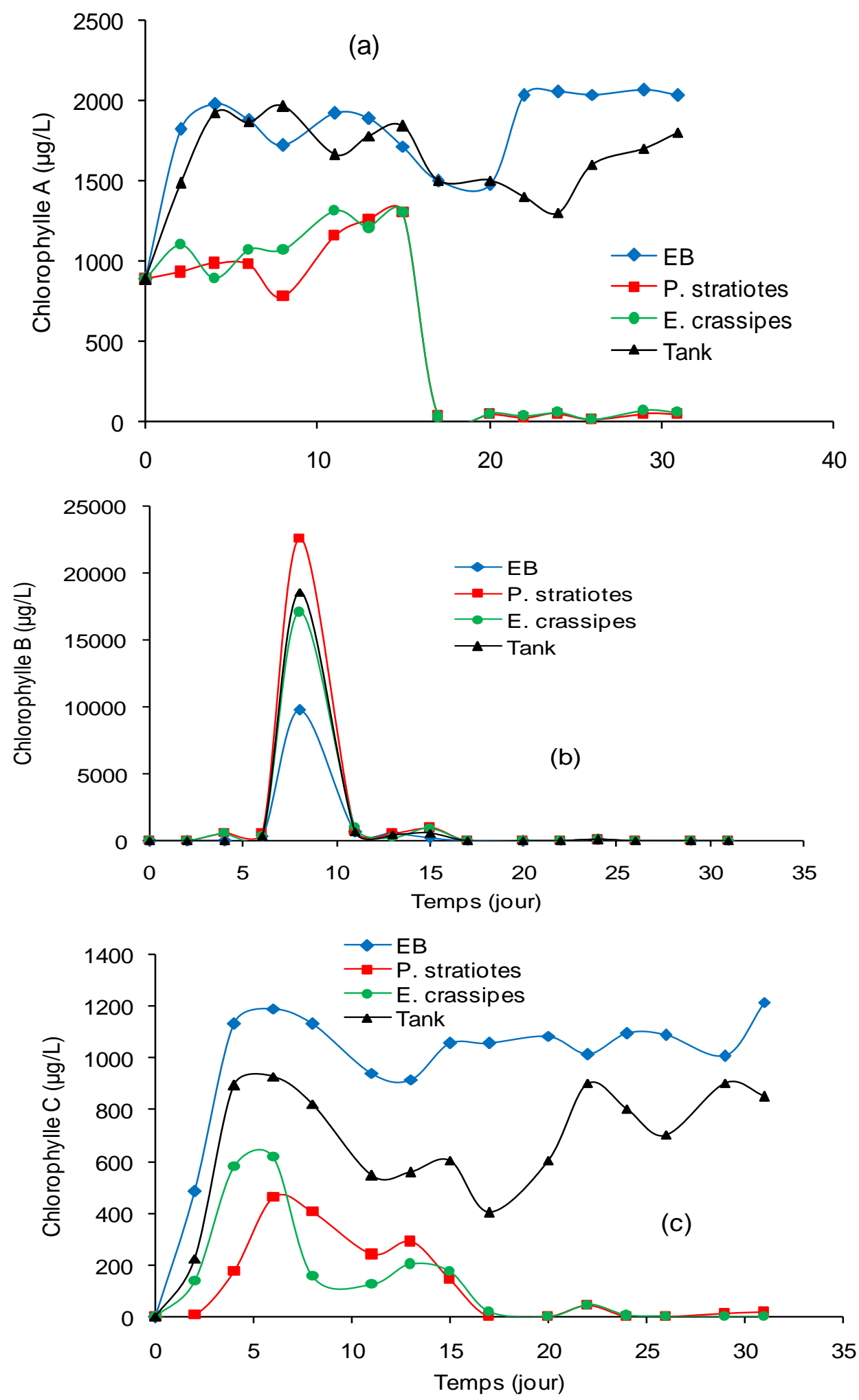

Figure 5 : Cinétique de réduction des Chlorophylles A(a), B(b) et (C) dans le dispositif expérimental. 
Tableau 2 : Taux d'abattement des paramètres de pollution.

\begin{tabular}{|c|c|c|c|c|c|c|c|}
\hline \multirow[b]{2}{*}{ Paramètres } & \multicolumn{2}{|c|}{$\begin{array}{c}\text { Effluent traité après } \\
15 \text { jours }\end{array}$} & \multicolumn{2}{|c|}{$\begin{array}{c}\text { Effluent traité après } \\
31 \text { jours }\end{array}$} & \multicolumn{2}{|c|}{$\begin{array}{l}\text { Effluent traité après } \\
31 \text { jours (Témoins) }\end{array}$} & \multirow{2}{*}{$\begin{array}{c}\begin{array}{c}\text { STEP de } \\
\text { Buterere }\end{array} \\
\begin{array}{c}\text { Valeur } \\
\text { finale } \\
(\mathrm{mg} / \mathrm{L})\end{array} \\
\end{array}$} \\
\hline & $\begin{array}{c}\text { Valeur } \\
\text { finale } \\
(\mathrm{mg} / \mathrm{L})\end{array}$ & $\begin{array}{c}Y \\
(\%)\end{array}$ & $\begin{array}{c}\text { Valeur } \\
\text { finale } \\
(\mathrm{mg} / \mathrm{L})\end{array}$ & $\begin{array}{c}Y \\
(\%)\end{array}$ & $\begin{array}{c}\text { Valeur } \\
\text { finale } \\
(\mathrm{mg} / \mathrm{L})\end{array}$ & $\begin{array}{c}Y \\
(\%)\end{array}$ & \\
\hline Chlorophylle A & 1,3044 & ++ & 0,0563 & 99,37 & 1,8056 & ++ & 6,125 \\
\hline Chlorophylle B & 0,9104 & ++ & 0,0395 & 93,77 & 0,0314 & ++ & 0,4243 \\
\hline Chlorophylle C & 0,1736 & ++ & 0,00 & 100,0 & 0,8503 & ++ & 1,2801 \\
\hline $\mathrm{PO}_{4}^{3-}$ & 18,94 & 32,61 & 15,11 & 46,21 & 21,71 & 29,45 & 25,40 \\
\hline $\mathrm{NH}_{4}^{+}$ & 7,37 & 91,10 & 11,52 & 86,08 & 38,0 & 54,10 & 68,50 \\
\hline $\mathrm{N}-\mathrm{NO}_{3}{ }^{-}$ & 10,74 & 57,36 & 45,76 & ++ & 23,72 & 6,27 & 41,20 \\
\hline DCO & 176,73 & 57,41 & 756,25 & ++ & 275,45 & 23,08 & 123,00 \\
\hline DBO & 63,33 & 75,64 & 66,66 & 74,36 & 110,00 & 57,69 & 51,00 \\
\hline
\end{tabular}

NB : ++ : concentration finale supérieure à la concentration initiale (absence d'abattement)

\section{DISCUSSION}

Le résultat d'élimination de $\mathrm{PO}_{4}{ }^{3-}$ autour de $46,21 \%$ est en accord avec celui rapporté par Rezania et al. (2016). Au cours de ce processus de phytoremédiation, l'élimination de $\mathrm{PO}_{4}{ }^{3-}$ au niveau du bassin à $E$. crassipes est moins importante que celui de $P$. stratiotes. Et pourtant, on s'attendrait à un abattement bien meilleur dans le deuxième bassin (E. crassipes) après un temps de séjour dans le premier bassin ( $P$. stratiotes). Cela pourrait s'expliquer par la fraction particulaire du phosphore qui minéralise dans le deuxième bassin sous l'action des bactéries (Jerbi, 2015) en libérant le phosphore soluble $\left(\mathrm{PO}_{4}{ }^{3-}\right)$. En outre, le bassin à E. crassipes reçoit aussi le phosphore résiduel issu du bassin à $P$. stratiotes. Cela entraîne une charge supplémentaire de $\mathrm{PO}_{4}{ }^{3-}$ à éliminer dans le deuxième bassin. Comme le phosphore, l'azote ammoniacal $\left(\mathrm{NH}_{4}{ }^{+}\right)$a été fortement éliminé pour satisfaire le besoin en nutriment des deux macrophytes. En effet, le processus biologique impliquant les bactéries, accélère la conversion de $\mathrm{NH}_{4}{ }^{+}$en $\mathrm{NO}_{3}{ }^{-}$par la réaction de nitratation (Equation 2) (Saeed and Sun, 2012). Ces bactéries pour assurer cette réaction, bénéficient de l'oxygène dissous issu de la photosynthèse des plantes qui ont pratiquement colonisé les plans d'eau. Cette couverture totale du plan d'eau maintient constant la température pour éviter toute variation de la teneur en oxygène dissous. C'est à juste titre, qu'au niveau des tanks témoins, une légère élimination de $\mathrm{NH}_{4}{ }^{+}$a été réalisée sous l'action des bactéries qui reçoivent seulement de l'oxygène à travers la photosynthèse des microphytes où l'équilibre thermique n'est point assuré. De façon générale, ce dispositif expérimental a permis d'avoir des résultats très intéressants au regard des travaux de Rezania et al. (2016), qui ont rapporté un taux d'élimination de $\mathrm{NH}_{4}{ }^{+}$autour de $85 \%$ de traitement en continu.

$$
\begin{aligned}
& \mathrm{NH}_{4}^{+}+2 \mathrm{O}_{2} \rightarrow \mathrm{NO}_{3}^{-}+\mathrm{H}_{2} \mathrm{O}+2 \mathrm{H}^{+}: \\
& \Delta G=-349,3 \mathrm{~kJ} / \mathrm{mol} \mathrm{N} \text { (Equation 2) }
\end{aligned}
$$

En ce qui concerne l'élimination du nitrate, après la chute de sa teneur correspondant à $57,36 \%$ de réduction au $15^{\mathrm{e}}$ jour, une remontée de cette teneur a été constatée au-delà de cette date car cette période correspond au pic de transformation biologique de $\mathrm{NH}_{4}{ }^{+}$en $\mathrm{NO}_{3}{ }^{-}$(conférer Figure 3). Ces résultats sont en accord avec les travaux antérieurs de ce que $E$. crassipes a une bonne capacité d'élimination de nitrate (Qin et al., 
2016; Victor et al., 2016).

Une évolution similaire de la teneur en chlorophylles dans les bassins témoins et dans les bassins à macrophytes a été remarquable. A travers les bassins témoins, on constate que le développement des macrophytes réduit la teneur en chlorophylles. Ces macrophytes sécrètent des composés phénoliques, des hydroquinones et de résorcinol ayant une activité inhibitrice à la croissance des microphytes responsables de la teneur en Chlorophylle (Ismail et al., 2015). En outre, la couverture intégrale du plan d'eau par les macrophytes empêche les rayons solaires d'activer la photosynthèse pour la croissance des microphytes. Ainsi, à partir du $15^{\mathrm{e}}$ jour, une chute de la teneur en Chlorophylles A a été remarquable au point d'atteindre parfois des valeurs nulles. Quant à la Chlorophylle $\mathrm{C}$, cette chute est décrite par une allure sinusoïdale avant d'atteindre le minima à zéro. Ces résultats sont en accord avec les travaux de Fang et al. (2007). Par contre, dans le bac témoin, l'absence d'une quelconque activité inhibitrice minimise la variation temporelle de la Chlorophylle A et un peu moindre celle de la Chlorophylle C. Pour la Figure 5b, on constate que c'est le contraire qui se produit. En effet, l'allure des courbes est la même, mais avec des concentrations en Chlorophylle B bien supérieures dans les bassins à macrophytes que dans l'eau brute. En d'autres termes, les substances inhibitrices n'ont pas d'influence sur les microphytes responsables de la teneur en Chlorophylles B. On observe une augmentation brusque de la teneur en chlorophylle $\mathrm{B}$ entre le $5^{\mathrm{ème}}$ et le $10^{\text {ème }}$ jour (Figure 5 (b)). Cette augmentation de la chlorophylle $\mathrm{B}$ serait due à la diminution de la chlorophylle A, principal pigment photosynthétique, entraînant ainsi l'inhibition de la photosynthèse consommatrice de la teneur de ce pigment. En effet, la chlorophylle B est le pigment accessoire qui collecte l'énergie et la transmet à la chlorophylle Ace qui signifie qu'en absence de la chlorophylle A, la chlorophylle B n'est pas utilisé par les macrophytes (Féret, 2009).

Enfin, l'élimination de la pollution organique évaluée par la DCO et la DBO
(Tableau 2) vient confirmer l'efficacité de ce dispositif expérimental à la dépollution des eaux usées domestiques. En effet, la DBO qui traduit la pollution organique biodégradable est restée plus ou moins constante depuis le $15^{\mathrm{e}}$ jusqu'au $31^{\mathrm{e}}$ jour avec un taux d'abattement autour de $74,36 \%$. Quant à la DCO qui traduit la présence des matières oxydables d'origine organique et minérale, un taux d'abattement d'environ $57,41 \%$ a été obtenu dès les deux premières semaines de traitement. Mais la minéralisation des matières dissoutes au cours de la phytoremédiation et la forte présence des nitrates ont été des facteurs favorisant l'augmentation de la DCO. Par conséquent, il est possible d'affirmer à travers la DBO que la pollution organique a connu une forte élimination par ces macrophytes à travers ce dispositif expérimental. Par ailleurs, au regard des résultats relatifs aux nutriments, une comparaison de la performance est en faveur de ce dispositif expérimental par rapport à la STEP de Buterere.

\section{Conclusion}

Cette étude a été entreprise pour traiter l'effluent des bassins facultatifs de la STEP de Buterere (Burundi) en simulant les conditions réelles par un pilote expérimental constitué des séries de tanks à $P$. stratiotes et $E$. crassipes en mode continu. A cet effet, la cinétique de la phytoremédiation des paramètres de pollution a été suivie à la sortie des tanks à $P$. stratiotes puis celle des tanks à E. crassipes. Cette étape a permis de comprendre que la réduction des paramètres est fonction de la croissance des macrophytes et de leur temps de séjour dans ces différents tanks à eau usée. Ainsi, certains paramètres comme les Chlorophylles $\mathrm{A}, \mathrm{B}$ et $\mathrm{C}$ ont été insensibles à la phytoremédiation car leurs concentrations n'ont cessé d'augmenter pour atteindre le pic au $15^{\mathrm{e}}$ jour. Mais après ce pic, une chute de la concentration des Chlorophylles (A, B et C) a été remarquable jusqu'au $31^{\mathrm{e}}$ jour car la couverture intégrale de la surface d'eau suite à une forte croissance des macrophytes a empêché le développement des microphytes. Cette croissance des macrophytes a été également favorable à l'élimination de l'azote ammoniacal, de phosphore et de la 
DBO avec des rendements respectifs de $86,08 \%, 46,21 \%$ et $74,36 \%$ au $31^{\text {e }}$ jour. Par contre, au cours de cette même période, une forte présence de nitrate et de la DCO a été constatée suite à la minéralisation des composés organiques et la nitrification de l'azote ammoniacal en nitrate. En effet, cette augmentation de la teneur en nitrate ne remet pas en cause la performance du dispositif expérimental. Cela doit nous amener à travers des études ultérieures, à mettre en place un troisième bassin en aval pour affiner le traitement tertiaire en utilisant soit $P$. stratiotes ou E. crassipes. Enfin, les résultats de cette étude devraient être appliqués pour substituer les bassins de maturation par les bassins à macrophytes afin d'améliorer l'efficacité des stations de lagunage de Buterere par exemple.

\section{CONFLIT D'INTÉRÊTS}

Tous les auteurs déclarent qu'il n'y a aucun conflit d'intérêts.

\section{CONTRIBUTIONS DES AUTEURS}

PN a participé à la conception du dispositif expérimental, à la collecte des échantillons et aux analyses effectuées au laboratoire. Il a participé à la rédaction du manuscrit. GN a participé à la conception du dispositif expérimental, à la mobilisation des financements et à la rédaction du manuscrit. BGHB et KA ont contribué à l'exploitation scientifique des données. DN et GNT ont contribué au processus de rédaction. Tous les auteurs ont lu et approuvé le manuscrit final.

\section{REMERCIEMENTS}

Cette étude a bénéficié des soutiens financiers de l'Ambassade de France au Burundi, du Projet VLIR-UOS/U.B, du Projet ARES Belgique et du Laboratoire de Chimie et d'Analyses Environnementales (LCAE) de l'Université du Burundi.

\section{REFERENCES}

AFNOR. 1994. Essais des Eaux. In: Qualité de l'eau. Association Française de normalisation, Paris.

Atinkpahoun C, Soclo H, Pons M-N, Leclerc J-
P.
2018.
Physico-chemical

characterization of domestic wastewaters in the "Vie-Nouvelle" agglomeration, Cotonou, Benin. International Journal of Biological and Chemical Sciences, 12(1): 542-557.

DOI: https://doi.org/10.4314/ijbcs.v12i1.42

Buhungu S, Montchowui E, Barankanira E, Sibomana C, Ntakimazi G, Bonou CA. 2018. Caractérisation spatio-temporelle de la qualité de l'eau de la rivière Kinyankonge, affluent du Lac Tanganyika, Burundi. International Journal of Biological and Chemical Sciences, 12(1): 576-595. DOI: https://doi.org/10.4314/ijbcs.v12i1.44

Buhungu S, Houssou AM, Montchowui E, Ntakimazi G, Vasel JL, Ndikumana T. 2017. Etablissement du pollutogramme et de l'hydrogramme de la rivière Kinyankonge, Burundi. International Journal of Biological and Chemical Sciences, 11(3): 1386-1399. DOI: https://doi.org/10.4314/ijbcs.v11i3.37

Carr RM, Blumenthal UJ, Duncan Mara D. 2004. Guidelines for the safe use of wastewater in agriculture: Revisiting WHO guidelines. Water Science and Technology, 50(2): $31-38$. DOI: https://doi.org/10.2166/wst.2004.0081

Fang YY, Yang XE, Chang HQ, Pu PM, Ding XF, Rengel Z. 2007. Phytoremediation of Nitrogen-Polluted Water Using Water Hyacinth. Journal of Plant Nutrition, 30(11): $\quad$ 1753-1765. DOI: https://doi.org/10.1080/15226510701375 507

Féret MJ-B. 2009. Apport de la modélisation pour l'estimation de la teneur en pigments foliaires par télédétection. PhD Thesis, Université Pierre ET Marie Curie, p. 203.

Gboko AJ, Akobe AC, Aka AM, Aka CA, Kouame AF, Adou KN, Yapo OB, Monde S, Aka K. 2019. Etat d'eutrophisation de la lagune continentale Ono (Bonoua sud-est de la Côte d'Ivoire) dans un environnement agro-industriel durant la crue du fleuve Comoé. International Journal of Biological and Chemical Sciences, 13(6): 2942-2958. 
DOI:

https://doi.org/10.4314/ijbcs.v13i6.40

Gupta P, Roy S, Mahindrakar AB. 2012.

Treatment of Water Using Water Hyacinth, Water Lettuce and Vetiver Grass: A Review. Resources and Environment, 2(5): 202-215. DOI: 10.5923/j.re.20120205.04

Ismail Z, Othman SZ, Law KH, Sulaiman AH, Hashim R. 2015. Comparative Performance of Water Hyacinth (Eichhornia crassipes) and Water Lettuce (Pista stratiotes) in Preventing Nutrients Build-up in Municipal Wastewater. CLEAN - Soil, Air, Water, 43(4): 521- 531.

DOI: https://doi.org/10.1002/clen.201200254

Jerbi NAG. 2015. Eutrophisation et dynamique du phosphore et de l'azote en Seine: Unnouveau contexte suite à l'amélioration du traitement des eaux usées. PhD Thesis, Université Pierre ET Marie Curie, p. 267.

Kone D. 2002. Epuration des eaux usées par lagunage à microphytes et à macrophytes en Afrique de l'ouest et du centre: État des lieux, performances épuratoires et critères de dimensionnement. These de doctorat, École Polytechnique Fédéralede Lausanne, p. 194.

Lu B, Xu Z, Li J, Chai X. 2018. Removal of water nutrients by different aquatic plant species : An alternative way to remediate polluted rural rivers. Ecological Engineering, 110: 18-26. DOI: https://doi.org/10.1016/j.ecoleng.2017.0 9.016

Mama D, Aina M, Alassane A, Boukari OT, Chouti W, Deluchat V, Bowen J, Afoud A, Baudu M. 2012. Caractérisation physico-chimique et évaluation du risque d'eutrophisation du lac Nokoué (Bénin). International Journal of Biological and Chemical Sciences, 5(5): 2076-2093. http://ajol.info/index.php/ijbcs

Mara D. 2003. Domestic Wastewater Treatment in Developing Countries (3rd ed). Earthscan: London, UK.

Mahunon S, Aina M, Akowanou AVO, Kouassi E, Yao B, Kopoin A, Drogui P.
2018. Optimization process of organic matter removal from wastewater by using Eichhornia crassipes. Environmental Science and Pollution Research, 25. DOI: https://doi.org/10.1007/s11356-0182771-y

Nsavyimana G. 2015. Modélisation des processus physiques et biologiques dans des fosses septiques et voies de valorisations des boues de Vidange: Application à Bujumbura-Burundi. Thèse de Doctorant, Université de Liège, p. 427

Qin H, Zhang Z, Liu M, Liu H, Wang Y, Wen X, Zhang Y, Yan S. 2016. Site test of phytoremediation of an open pond contaminated with domestic sewage using water hyacinth and water lettuce. Ecological Engineering, 95: 753-762. DOI:

https://doi.org/10.1016/j.ecoleng.2016.0 7.022

Rezania S, Din MFM, TaibS M, Dahalan FA, Songip AR, Singh L, Kamyab H. 2016. The efficient role of aquatic plant (water hyacinth) in treating domestic wastewater in continuous system. International Journal of Phytoremediation, 18(7): 679- 685.

DOI: https://doi.org/10.1080/15226514.2015.1 130018

Saeed T, Sun G. 2012. A Review on Nitrogen and Organics Removal Mechanisms in Subsurface Flow Constructed Wetlands : Dependency on Environmental Parameters, Operating Conditions and Supporting Media. Journal of Environmental Management, 112: 429-448. DOI: https://doi.org/10.1016/j.jenvman.2012.0 8.011

Shah M, Hashmi HN, Ghumman AR, Zeeshan M. 2015. Performance assessment of aquatic macrophytes for treatment of municipal wastewater. Journal of the South African Institution of Civil Engineering, 57(3): 18-25. DOI: https://doi.org/10.17159/23098775/2015/V57N3A3

Sindayigaya S. 2015. Républiquedu Burundi, Ministère de l'Eau, de l'Environnement, 
de l'Aménagement du Territoire et de l'Urbanisme. Rapport National pour Habitat III : Programme des Nations Unies pour le Développement, p. 71.

Ting WHT, Tan IAW, Salleh SF, Wahab NA. 2018. Application of water hyacinth (Eichhornia crassipes) for phytoremediation of ammoniacal nitrogen: A review. Journal of Water Process Engineering, 22: 239- 249. DOI: https://doi.org/10.1016/j.jwpe.2018.02.0 11

Ugya AY, Hua X, Ma J. 2019. Phytoremediation as a tool for the remediation of wastewater resulting from dyeing activities. Applied Ecology and Environmental Research, 7, 3723-3735. DOI:

https://doi.org/10.15666/aeer/1702_3723 3735

Victor KK, Séka Y, Norbert KK, Sanogo TA, Celestin AB. 2016. Phytoremediation of wastewater toxicity using water hyacinth (Eichhornia crassipes) and water lettuce (Pistia stratiotes). International Journal of Phytoremediation, 18(10): 949-955.
DOI:

https://doi.org/10.1080/15226514.2016.1 183567

Wang CY, Sample DJ. 2014. Assessment of the nutrient removal effectiveness of floating treatment wetlands applied to urban retention ponds. Journal of Environmental Management, 137: 23-35. DOI: https://doi.org/10.1016/j.jenvman.2014.0 2.008

Yahiaoui K, Zoubeidi A, Rouahna N, Ouakouak A. 2018. Study of domestic wastewater treatment bymacrophyte plant in Arid Region of South-east Algeria (case of el oued region). Journal of Fundamental and Applied Sciences, 10(2): Article 2. DOI: https://doi.org/10.4314/jfas.v10i2.19

ZimmelsY, Kirzhner F, Malkovskaja A. 2007. Application of Eichhornia crassipes and Pistia stratiotes for treatment of urban sewage in Israel. Journal of Environmental Management, $\mathbf{8 1}$ : 420- $428 . \quad$ DOI: https://doi.org/10.1016/j.jenvman.2005.1 1.014 . 